\title{
Challenges in Designing New Interfaces for Musical Expression
}

\author{
Rodrigo Medeiros ${ }^{1}$, Filipe Calegario ${ }^{1}$, Giordano Cabral $^{2}$, Geber Ramalho $^{1}$ \\ ${ }^{1}$ Centro de Informática, Universidade Federal de Pernambuco, Av. Prof. Luis Freire, s/n, \\ Cidade Universitária. Recife - PE, CEP: 50740-540, Brasil \\ ${ }^{2}$ Departamento de Estatística e Informática, Universidade Federal Rural de Pernambuco, Rua \\ Dom Manuel de Medeiros, s/n. Dois Irmãos. Recife - PE, CEP: 52171-900, Brasil \\ proferodrigomedeiros.com.br, fcacecin.ufpe.br, \\ giordanorecegmail.com, glrecin.ufpe.br
}

\begin{abstract}
The new interfaces are changing the way we interact with computers. In the musical context, those new technologies open a wide range of possibilities in the creation of New Interfaces for Musical Expression (NIME). Despite 10 years of research in NIME, it is hard to find artifacts that have been widely or convincingly adopted by musicians. In this paper, we discuss some NIME design challenges, highlighting particularities related to the digital and musical nature of these artifacts, such as virtuosity, cultural elements, context of use, creation catalysis, success criteria, adoption strategy, etc. With these challenges, we aim to call attention for the intersection of music, computing and design, which can be an interesting area for people working on product design and interaction design.
\end{abstract}

Keywords: design challenges, digital musical instrument, new interfaces for musical expression, user experience, interaction design.

\section{Introduction}

The whole way we interact with machines is changing, since gestures, movements and direct graphic manipulation are co-existing with keys, buttons and pointers $[1,2]$. In the musical context, these new interface technologies open a wide range of possibilities in the creation of Digital Musical Instruments (DMI) or New Interfaces for Musical Expression (NIME), artifacts that connect inputs (interface controllers) and outputs (sound synthesis modules) according to a mapping strategy [3].

Despite 10 years of research in NIME, reported in conferences (such as New Music for Music Expression, and International Computer Music Conference) and journals (such as Organised Sound, Journal of New Music Research and Computer Music Journal), it is hard to find artifacts that have been widely or convincingly adopted by musicians. In fact, there are very few NIME virtuosi or professional musicians who adopted them as their main musical instrument. Few exceptions of commercial success are the artifacts related to electronic music, DJ interfaces and controllers.

adfa, p. 1, 2011.

(C) Springer-Verlag Berlin Heidelberg 2011 
We believe there are some design challenges that may help to explain the state of the art in NIME research and practice, which are not well described, analyzed together or explicitly discussed in the literature. Topics such as usability, efficiency or fun are not obvious when applied to new musical instruments design, indeed.

This paper aims to discuss some challenges related to the design and the development of NIME, which presents a series of particularities due to their digital and musical nature. In fact, some issues are specially hard, such as how to deal with virtuosity, how to include cultural elements surrounding the artifact, how to consider the musician context in his/her experience in using the artifact, how to catalyze the creation of new artifacts, how to define what is a successful design, how to promote adequately the adhesion of adopters, etc.

We hope that, enumerating these interesting challenges, this paper will instigate people working in product design and interaction design, particularly in user-centered design and user experience (UX), to pay more attention this intersection of music, computing and design. We believe that HCI studies can provide important principles, methods and techniques for NIME development. In turn, we will try to show that HCI domain can take advantage from NIME design challenges, beyond the music domain [4].

In order to make its scope clearer, we would like to stress that this paper discusses musical instruments as artifacts to be used by professional musicians in all their potential. We are not interested in discussing the design of musical toys, artifacts created to make the experience of playing music easier for a non-professional public [5]. Even though musical toys may be important in some contexts, such as for musical initiation, promotion and education, they do not present the same design challenges.

The next section presents the definition and some examples of NIME. The following sections present some challenges on how to design NIME, divided into two parts: Section 3, the challenges of being digital, and Section 4, the challenges of being musical.

\section{DMI and NIME}

A digital musical instrument "consists of a control surface or gestural controller, which drives the musical parameter of a sound synthesizer in real time" [3]. It can be separated in three parts: (a) the input or gestural control; (b) the output or sound synthesis; and (c) the mapping strategies between input and output. In literature, some authors make distinctions between DMI and NIME definitions. However, it is not a consensus. In this paper, this distinction is not relevant for our main discussion and we assume that both definitions are synonymous.

These new musical interfaces can be classified by the resemblance to existing acoustic instruments: augmented musical instruments, instrument-like gestural controllers, instrument-inspired gestural controllers, alternate gestural controllers [3]. It is a diverse universe of artifacts with different natures. The following examples show the range of this variety: the hyperinstruments [6], AKAI EWI [7], Yamaha WX5, 
Hands [3], Radio Baton [8], Reactable [9], Laser Harp, illusio [10], Tenorion [11], Novation Launchpad, Monome, Faderfox, and QuNeo [12].

As the physical constraints of acoustic instrument do not exist in NIME, the design of these new interfaces are more flexible concerning the coupling between input gesture and generated sound. Therefore, new ways of producing live music arise and go beyond the traditional concept of musical instrument which focused on controlling how notes are played [12]. NIME extends note control to process control, e.g. with simple gestures, the musician can trigger not only set of notes, but pre-recorded samples, loops, sound effects, etc. Consequently, the performer engages in different activities when playing those new interfaces: create, when interacting with notes and the sound synthesis parameters and control, when triggering processes as samples, loops [12].

\section{$3 \quad$ Challenges of Being Digital}

In this section, we enumerate some problems faced when designing digital musical instruments, compared to when designing acoustic instruments [13]. Identifying these problems is important to better understand the challenges discussed in Section 4.

\subsection{The Mapping Problem}

Contrary to acoustic instruments, which impose physical constraints to their design and fabrication regarding the connection of the input (possible gestures) to the output (sound generation), the design of NIME has more freedom. Due to the separation between gesture input and sound output, the mapping strategies are the essence of digital musical instruments [14]. Different mapping strategies for the same set of inputs and outputs affect how the performer reacts musically and psychologically to the instrument [15]. Those strategies are virtually countless [14]. Paradoxically, this advantage is a problem, since there is no established method or tool to guide the NIME designer to define how interfaces gestures should be adequately mapped into sound variables [16].

\subsection{Audience Understanding and Engagement}

Strongly related to the mapping problem is how does the audience understand the performance? Since the gestures and the produced sound have not necessarily a direct connection, the audience may not understand what is happening, and consequently, does not feel engaged during the appreciation [5]. If the audience is expecting something related to a musical instrument, they can get frustrated by the lack "observable primary causation" [12]. In the literature, this is called transparency, which can be defined as "the psychophysiological distance, in the player and the audience minds, between the input and output of a device mapping" [17]. In short, the creation of meaningful and perceivable connections between human action and sound is not only an issue for the musicians, but also for the audience [18]. There are some possible 
solutions for this problem, such as the use of well-designed visual feedback; visual cues as instrument's appearance, visualization of interaction and visualization of sound output; interaction metaphors [5]. However, this problem remains opened.

\subsection{Sensor Limitations}

All design projects have to deal with technical limitations and musical instruments is not an exception. Specifically, it's needed to take into account sensors and actuators limitations. The limitations comprise three subtypes: real time (latency and jitter), precision (the $\mathrm{AD}$ conversion requires the quantization of continuous quantities. Thus, the granularity and the way information is represented may be a problem), and information richness (some important data may be ignored by sensors).

\subsection{Embodied Relationship and Haptic Feedback}

In acoustic instruments, the excitation of notes and the sound generation are intrinsically linked. With the dissociation of these two components, the musician can explore limitless possibilities. However, he/she loses a strong relation with the concrete body of the instrument, what Payne describes as an embodied relationship [12]. For instance, "A pianist can see and locate a specific key before playing it, can use the resistance of the key-action mechanism to help know how hard to press the key, and can use the feeling of adjacent keys to keep track of hand position" [19]. NIME design must face how to provide haptic feedback to the performer.

\section{$4 \quad$ Challenges of Being Musical}

In this section, we enumerate some challenges involved in designing digital artifacts that we be used to create and play music.

\subsection{Expressivity and Virtuosity}

Probably the most important artistic property of a musical instrument (digital or not) is to enable the player to be expressive, i.e. to "effectively convey meaning or feeling" [20]. Traditionally, music expressiveness is coded by musicians through a subtle control over some sonic attributes of individual notes and musical phrases, such as timing, volume, timbre, accents, and articulation [19]. Artistic expressivity is also required for instruments oriented to process control rather than notes, which is the case of DJs performances using a turn-table, for instance.

As a consequence, an obvious challenge in designing a musical instrument is to enable sophisticated, fine-grained and subtle control of input gestures with their correspondence in sound output [21]. Indeed, it is not enough to build an instrument that plays notes. It should enable to play notes with a particular timbre, intonation, intensity, etc. This requirement make all the problems discussed in the previous section harder [19]. The sensors must deal with a large amount of subtle gestures, the sound 
synthesis must include the control of various sonic parameters, and the I/O mapping must deal with one-to-many, many-to-one, and many-to-many mappings and combinations of parameters.

These are other challenges in designing NIME as a result of the expressiveness need. In fact, musical expression requires not only an excellent control interface, but also virtuosic mastery of the instrument by the human player. Virtuosity is that "great technical skill" [20] that enables the player to master so well the subtle controls of the instrument that he/she can perform other cognitive activities as the music interpretation [22]. The lack of virtuosity may inhibit music expression, since a virtuoso does not only successfully realize a highly difficult task, but he/she does it expressively [23].

Mastering musical instruments demands years (some authors claim 10 years [24]) of studying and practicing. In other words, professional musicians are ready to spend hours in a daily-basis trying to master an interface that was created more to be expressive than to be easily usable [25]. Some instruments might even "hurt" new users (e.g., fingers of a beginner guitar player, or the lips for a trumpet player), but they still go on.

In terms of designing new instruments, a consequence of this concerns the evaluation of the artifact, which is an essential activity in the development process [26, 27]. If the artifact is supposed to be used by a virtuoso, how to know that an identified execution problem is a design error or a natural barrier that would be overcame by training? How the musician evolution could be taken into account in the evaluation protocol of the artifact? The concept of usability or UX in the context of NIME may be far trickier than we are used to discuss in most of HCI studies.

Another consequence of the "virtuosity factor" concerns user adoption. How to convince and favor people to engage in experimentation a new artifact that will demand huge amount of hours of dedication? Is there any kind of reward strategy that could be useful for motivating adopters? This problem is even harder in designing NIME, because contrary to conventional acoustic instruments, there is no virtuoso demonstrating how far one can go with a given instrument, which establishes somehow a standard.

Moreover, there is almost no musical repertoire created to draw the attention of potential adopters or to contribute to the technical advances of the instrument [25]. Learning a conventional instrument is helped by a body of technical knowledge, created over dozens of years (or centuries) and transmitted by teachers, books, concerning how to efficiently practice the instrument. How to be sure that the adopters are practicing is the most effective and efficient way, given that the instrument is entirely new? How to create a new instrument, its musical repertoire and its studying method in the same time stamp?

\subsection{Context of Use and Stakeholders}

Another important challenge is that the designer must be aware of the context in which the instrument will be played, since it may influence the musician satisfaction, and, consequently, the instrument adoption and refinement $[12,28]$. 
There are different contexts of use. For instance, a performer can play solo or in a group. Also, he/she can improvise, play a predefined score or accompany others. There are equally different mindsets depending on the activity the musician is doing: studying, rehearsing, or facing an audience. Each context may demand different properties from the instrument. For example, playing with others may demand a minimum level of volume for the instrument to be listened; the requirement of playing an established repertoire may lead to a tonal instrument; etc.

The design process is harder when one needs to deal with multiple contexts that may substantially influence the musician experience of use. How to conceive an instrument which simultaneously serves to different purposes such as make solos, to accompany, to be played collaboratively, to compose, to perform before an audience? How to identify the needs of each context and how to take into account the different contexts characteristics in the design process?

Another important challenge related to context of use is the stakeholders' point of view. According to Payne, "any implementation of a new musical interface must therefore consider the ecology of this environment" [12]. There are different perspectives and stakeholders, such as the audience, the performer, the composer, the designer and the manufacturer $[18,28]$, involved in NIME design and use.

As discussed in the section 3.2, meaningful connections between musician actions and the generate sound should be provided to the audience. The emotional exchange and communication between the performer and the audience is an important factor in the performer satisfaction. Thus, if the audience did not like the performance because NIME was not understandable or exciting, this will influence the instrument adoption by the musician. How to incorporate coherently in the design process the stakeholders' perspectives? In particular, given that user experience is normally studied between the user and the artifact, how to go from the user-artifact binomial to an userartifact-audience tripod?

\subsection{Evolution and Success Criteria}

One of the components of a design process is to be able to evaluate if the designed artifact is successful or not $[29,30]$. This raises two questions: What defines success of NIME? How to incorporate success measures in the NIME design process?

The discussion of criteria success for NIME is not mature enough. This is probably due to the fact that lots of NIME are created to be used by the very creator [31]. Contrary to HCI community, where it is clear that artifacts or experiences must be designed to be used by someone else [2, 26, 29], the observation of some basic design principles is not always done by NIME practitioners or researchers.

We can group the success criteria discussed in the literature into two categories. The first one concerns the intrinsic measurement of quality, i.e. how the artifact fulfilled some requirements related to ergonomics, sound quality, visual feedback, finegrained gesture control, embodied relationship, ease of use, efficiency, learning curve, etc. [31]. However, which are the most important criteria? How does the context or stakeholder perspective influence the criteria choice? Is it enough to fulfill these requirements to guarantee NIME adoption? 
The second category includes various criteria (adhesion, impact, lifetime, commercial success, etc.), all of them requiring long time to be measured. This represents already a challenge, since we need the criteria during the development phase. However, even on the long-term, these criteria may be not objective enough.

One can say that an instrument has a high adhesion when it is adopted by a wide audience, when it is popular or it had a commercial success (number of units sold, sales volume or growth). The problem with this notion is that some are vocationally 'generic' and others highly specialized [32]. A specialized instrument may do not sell a lot, but be a success to a particular musician community.

The impact of an instrument is understood as how it has helped to create new music genres, new playing styles outstanding artworks [23]. The problem is that this takes a lot of time, cannot be planned in advance and depends on lots of variables, including cultural ones.

Persistence or lifetime is indicated as the most trustable measure of musical instruments success, Paine [33], for instance, says that "if we agree that acoustic instruments are 'successful' interfaces for music making, an assumption supported by the period of time they have persisted and the ubiquitous nature of traditional interfaces $(. .)$.$" .$

In fact, the evolution of acoustic instruments is based on trial-and-error process that can take centuries [34]. Therefore, the results about the design process can only be evaluated after very long periods of time. For a demanding and growing area, a relevant challenge is how this process can be accelerated. How can we decrease a 100 -years process into a, for example, 2-years one? Can an iterative process with short iterations and with an intense relation with artists and early adopters (usercentered design [26]) be a productive approach to achieve shorter instrument design processes?

\subsection{Beyond the Artifact}

Another basic challenge is that music is enveloped in a culture, sometimes bringing semantics or symbolism. For example, it is common to find musical instruments deeply related to their original region, nation, or people, as well as to particular attitudes. In other words, the musical instrument is more than just the artifact. It invokes the cultural elements that surround it. Thus, it is important to understand not only the conjuncture in which the instrument is inserted, but the strategies of adhesion.

For example, the adhesion of an instrument may be deeply related to a musical repertoire produced with it. "Some started playing after having been inspired by some music and, in particular, the sound of the instrument" [35]. There is a virtuous cycle related to the repertoire, the demonstrations and the instrument. Demonstrations need a repertoire, a set of compositions that can be played with the instrument. These compositions can even transmute into a new style. With demonstrations, potential musicians can understand what can be done with the instrument and engage in learning it.

For instance, let's take a look at the electric guitar and the Rock n'Roll. It's easy to imagine what can be done with an electric guitar by listening to some rock songs and watching some performances of rock musicians. This can attract new players and 
those new players, as they play the instrument, can go further in generating new repertoire and playing techniques.

The actual problem is that these strategies are frequently off the hands of the designer. What can a designer do if the success of a NIME highly depends on factors related to more than just the artifact? It won't be better if designers practice in planning marketing strategies? Even if this is often a responsibility of other professional, there are marketing strategies specific for musical instruments and designers could take advantage of it. In summary, it is important to know all the possible adhesion strategies, since it may provide shortcuts to a more successful instrument.

Shouldn't designers be closer to the final artists? For example, inviting composers to test the instrument may be good to generate a repertoire for it. Working together with an artist in a piece may also be very important, because "without a piece, it's difficult to gain acceptance" [5].

\section{Conclusions}

In this paper, we enumerate a series of design challenges for NIME that have not yet been systematically organized or deeply addressed in the literature. With the growth of the demand and the possibilities of building new interfaces for music expression, we believe that having those challenges more clearly stated, we can go a step towards better artifacts and more engaging user experience.

We believe that HCI expertise can improve NIME development. In turn, HCI can also take advantage from NIME area. The challenges presented here are a fertile research field and can provide fruitful insights for product and interaction design outside the musical domain: artifacts that requires a long time to acquire skill, that deals with different contexts of use, whose stakeholders play an important role, and whose use is deeply influenced surrounding culture, community, marketing, repertoire, etc.

As future work, we consider to deepen the relation between HCI and NIME by systematically discussing the crossover between HCI tools, techniques and processes with the NIME design challenges presented in this paper.

Acknowledgements. This research is partially funded by CAPES and CNPq.

\section{References}

1. Saffer, D.: Designing Gestural Interfaces. O’Reilly Media, Inc. (2008).

2. Moggridge, B., Smith, G.C.: Designing interactions. The MIT Press (2007).

3. Miranda, E.R., Wanderley, M.M.: New Digital Musical Instruments: Control and Interaction Beyond the Keyboard. A-R Editions, Middleton (2006).

4. Wallis, I., Ingalls, T., Campana, E., Vuong, C.: Amateur Musicians, Long-Term Engagement, and HCI. In: Holland, S., Wilkie, K., Mulholland, P., and Seago, A. (eds.) Music and Human-Computer Interaction. pp. 49-56. Springer-Verlag (2013).

5. Lyons, M., Fels, S.: Creating new interfaces for musical expression. SIGGRAPH Asia 2013 Courses on - SA ’13. pp. 1-164. ACM Press, New York, New York, USA (2013). 
6. Machover, T.: Hyperinstruments: A composer's approach to the evolution of intelligent musical instruments. Organ. Sound. 67-76 (1991).

7. Vashlishan, M.J.: The Akai Electric Wind Instrument (EWI4000s): A Technical and Expressive Method, (2011).

8. Mathews, M.: Radio Baton, http://www.csounds.com/mathews/, (2005).

9. Jordà, S., Geiger, G., Alonso, M., Kaltenbrunner, M.: The reacTable: exploring the synergy between live music performance and tabletop tangible interfaces. Proceedings of the 1st international conference on Tangible and embedded interaction (2007).

10. Barbosa, J., Calegario, F., Teichrieb, V.: Illusio: A Drawing-Based Digital Music Instrument. Proceedings of the International Conference on New Interfaces for Musical Expression (2013).

11. Nishibori, Y., Iwai, T.: Tenori-on. Proceedings of the International Conference on New Interfaces for Musical Expression. pp. 172-175. IRCAM - Centre Pompidou (2006).

12. Paine, G.: New Musical Instrument Design Considerations. MultiMedia, IEEE. 76-84 (2013).

13. Magnusson, T., Mendieta, E.: The acoustic, the digital and the body: A survey on musical instruments. Proc. Int. Conf. New Interfaces Music. Expr. 94-99 (2007).

14. Rovan, J.B., Wanderley, M.M., Dubnov, S., Depalle, P.: Instrumental gestural mapping strategies as expressivity determinants in computer music performance. Proceedings of Kansei-The Technology of Emotion Workshop. pp. 3-4. Citeseer (1997).

15. Hunt, A., Wanderley, M.M., Paradis, M.: The Importance of Parameter Mapping in Electronic Instrument Design. J. New Music Res. 32, 429-440 (2003).

16. Calegario, F., Barbosa, J., Ramalho, G., Cabral, G., Finch, G.: Sketchument: Empowering users to build DMIs through prototyping. Organised Sound. 18, 314-327 (2013).

17. Murray-Browne, T., Mainstone, D., Bryan-Kinns, N., Plumbley, M.D.: The medium is the message: Composing instruments and performing mappings. Proceedings of the International Conference on New Interfaces for Musical Expression. pp. 56-59 (2011).

18. O'Modhrain, S.: A framework for the evaluation of digital musical instruments. Comput. Music J. 35, 28-42 (2011).

19. Dobrian, C., Koppelman, D.: The E in NIME: Musical Expression with New Computer Interfaces. Proceedings of the International Conference on New Interfaces for Musical Expression. pp. 277-282 (2006).

20. Merriam-Webster: Merriam-Webster's collegiate dictionary. Merriam-Webster (2004).

21. Jordà, S.: Digital Instruments and Players : Part II - Diversity, Freedom and Control. Proceedings of the 2004 International Computer Music Conference (2004).

22. Hunt, A., Wanderley, M.M., Kirk, R.: Towards a Model for Instrumental Mapping in Expert Musical Interaction. Proceedings of the 2000 International Computer Music Conference. pp. 209-212 (2000).

23. Gurevich, M., Stapleton, P., Bennett, P.: Designing for style in new musical interactions. Proceedings of the International Conference on New Interfaces for Musical Expression. pp. 213-217 (2009).

24. Wanderley, M.M., Orio, N.: Evaluation of Input Devices for Musical Expression: Borrowing Tools from HCI. Comput. Music J. 26, 62-76 (2002).

25. Oore, S.: Learning advanced skills on new instruments. Proceedings of the International Conference on New Interfaces for Musical Expression. pp. 60-64.

26. Preece, J., Rogers, Y., Sharp, H.: Interaction Design: Beyond Human-Computer Interaction. Wiley (2002).

27. Laurel, B.: Design research: Methods and perspectives. the MIT Press (2003). 
28. Kvifte, T., Jensenius, A.: Towards a coherent terminology and model of instrument description and design. Proceedings of the International Conference on New Interfaces for Musical Expression. pp. 220-225 (2006).

29. Buxton, B.: Sketching User Experiences: Getting the Design Right and the Right Design. Morgan Kaufmann (2010).

30. Lowgren, J., Stolterman, E.: Thoughtful interaction design: A design perspective on information technology. The MIT press (2004).

31. Jordà, S.: Digital Lutherie, http://dialnet.unirioja.es/servlet/tesis?codigo=19509, (2005).

32. Jordà, S.: Digital instruments and players: part I---efficiency and apprenticeship. Proc. Int. Conf. New Interfaces Music. Expr. 59-63 (2004).

33. Paine, G.: Towards Unified Design Guidelines for New Interfaces for Musical Expression. Organised Sound. 14, 142 (2009).

34. Sachs, C.: The History of Musical Instruments. W. W. Norton \& Company, Inc. (1940).

35. Green, L.: How Popular Musicians Learn: A Way Ahead for Music Education. Ashgate Publishing (2002). 